\title{
Nobel Lecture: The BEH mechanism and its scalar boson ${ }^{*}$
}

\author{
François Englert ${ }^{\star}$ \\ Service de Physique Th orique, Universit Libre de Bruxelles, Campus Plaine C.P. 225, \\ Boulevard du Triomphe, B-1050 Bruxelles, Belgium \\ and The International Solvay Institutes, Campus Plaine C.P. 231, Boulevard du Triomphe, \\ B-1050 Bruxelles, Belgium
}

(published 3 July 2014)

\section{CONTENTS}

I. Introduction: Short and long range interactions

II. Spontaneous Symmetry Breaking

A. Spontaneous symmetry breaking in phase transitions

B. Spontaneous symmetry breaking in field theory

III. The BEH Mechanism

A. The fate of the Nambu-Goldstone boson and vector boson masses

B. The fate of the massive scalar boson

C. Fermion masses

D. Why is the mechanism needed?

E. Dynamical symmetry breaking

F. The electroweak theory and the standard model

IV. The Discovery

References

\section{INTRODUCTION: SHORT AND LONG RANGE INTERACTIONS}

Physics, as it is conceived today, attempts to interpret the diverse phenomena as particular manifestation of testable general laws. Since its inception at the Renaissance, mainly by Galileo's revolutionary concepts, this has been an extraordinary successful adventure. To the point that after impressive developments in the first half of the twentieth century, one might have even conceived that all phenomena, from the atomic scale to the edge of the visible Universe, be governed solely by two fundamental laws, and two known laws. Namely, classical general relativity, Einstein's generalization of Newtonian gravity, and quantum electrodynamics, the quantum version of Maxwell's electromagnetic theory.

Gravitational and electromagnetic interactions are long range interactions, meaning they act on objects no matter how far they are separated from each other. The progress in the understanding of such physics applicable to large scales is certainly tributary to the fact they can be perceived without the mediation of highly sophisticated technical devices. But the discovery of subatomic structures had revealed the existence of other fundamental interactions that are short range, that is negligible at larger distance scales. In the beginning of the 1960s, there was no consistent theoretical interpretation of short range fundamental interactions, neither of the "weak interactions" responsible for radioactive decays, nor of the

*The 2013 Nobel Prize for Physics was shared by François Englert and Peter Higgs. These papers are the text of the address given in conjunction with the award.

*fenglert@ulb.ac.be "strong interactions" responsible for the formation of nuclear structures.

Robert Brout and I (Englert and Brout, 1964), and independently Peter Higgs (Higgs, 1964a), constructed a mechanism to describe short range fundamental interactions. Robert Brout passed away in 2011 and left me alone to tell our story. I will explain how we were led to propose the mechanism, how it allows for consistent fundamental theories of short range interactions and for building elementary particle masses. It became a cornerstone of the standard model and was recently confirmed by the magnificent discovery at CERN of its predicted scalar boson.

We became convinced that a consistent formulation of short range interactions would require a common origin for both short and long range interactions.

While both classical general relativity and quantum electrodynamics describe long range interactions and are both built upon very large symmetries, labeled "local symmetries," they have very different structures: in contradistinction to general relativity, the long range quantum electrodynamics is fully consistent at the quantum level and was experimentally verified at that level, in particular, by the successful inclusion of chemistry in the realm of known physics. As a valid theory of short range interactions clearly required quantum consistency, we were naturally driven to take, as a model of the corresponding long range interactions, the generalization of quantum electrodynamics, known as Yang-Mills theory.

The quantum constituents of electromagnetic waves are "photons," massless neutral particles traveling with the velocity of light. Their massless character implies that the corresponding waves are polarized only in directions perpendicular to their propagation. These features are apparently protected by the local symmetry, as the latter does not survive the explicit inclusion of a mass term in the theory. Yang-Mills theory is built upon similar local symmetries, enlarged to include several massless interacting quantum constituents, neutral, and charged ones. These massless objects are labeled gauge vector bosons (or often simply gauge bosons).

To transmute long range interactions into short range ones in the context of Yang-Mills theory it would suffice to give these generalized photons a mass, a feature that, as we just indicated, is apparently forbidden by the local symmetries. Leaving momentarily aside this feature, let us first recall why massive particles transmit in general short range interactions.

Figure 1 is a Feynman diagram whose intuitive appearance hides a precise mathematical content. Viewing time as running from bottom to top, it describes the scattering of two electrons 


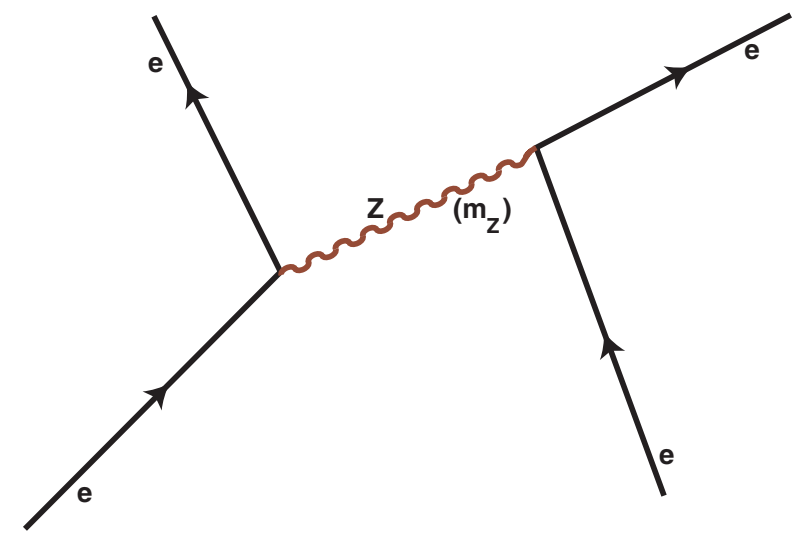

FIG. 1 (color). Massive particle mediating short range interactions.

resulting from the exchange of a massive particle labeled $Z$ of mass $m_{Z}$. Classically such a process could not occur as the presence of the $Z$ particle would violate energy conservation. Quantum mechanically it is allowed if the violation takes place within a time span of the order $\hbar / m c^{2}$. This process then describes in lowest order perturbation theory a short range interaction cutoff at a range $\sim \hbar / m c$.

As local symmetries apparently prevent the introduction of massive gauge bosons in the theory, we turn our attention to a class of theories where the state of a system is asymmetric with respect to the symmetry principles that govern its dynamics. This is often the case in the statistical physics of phase transitions (Launda, 1937). This is not surprising since more often than not energetic considerations dictate that the ground state or low lying excited states of a many body system become ordered. A collective variable such as magnetization picks up expectation value, which defines an order parameter that otherwise would vanish by virtue of the symmetry encoded in the formulation of the theory (isotropy in the aforementioned example). This is an example of spontaneous symmetry breaking (SSB) which frequently occurs in the statistical theory of second order phase transitions. Could the mass of gauge bosons arise through a similar SSB? This question arises naturally from the seminal work of Nambu who showed that SSB could be transferred from the statistical theory of phase transitions to the realm of relativistic quantum field theory (Nambu, 1960a, 1960b; Nambu and Jona-Lasinio, 1961a, 1961b), the mathematical framework designed to analyze the world of elementary particles.

This raises a deeper question: could SSB be the agent of the transmutation of long range interactions mediated by massless gauge fields to short range interactions mediating by massive ones without impairing the validity of the quantum behavior that characterize the simplest Yang-Mills theory, namely, quantum electrodynamics?

As we shall see the answer is yes to both questions provided the notion of SSB is traded for a more subtle one: the Brout-Englert-Higgs (BEH) mechanism (Englert and Brout, 1964; Higgs, 1964a). To prepare for the discussion of the mechanism, I will first review how SSB can be transferred from the theory of phase transitions to relativistic quantum field theory.

\section{SPONTANEOUS SYMMETRY BREAKING}

\section{A. Spontaneous symmetry breaking in phase transitions}

Consider a condensed matter system, whose dynamics is invariant under a continuous symmetry. As the temperature is lowered below a critical one, the symmetry may be reduced by the appearance of an ordered phase. The breakdown of the original symmetry is always a discontinuous event at the phase transition point but the order parameters may set in continuously as a function of temperature. In the latter case the phase transition is second order. Symmetry breaking by a second order phase transition occurs, in particular, in ferromagnetism, superfluidity, and superconductivity.

I first discuss the ferromagnetic phase transition which illustrates three general features of the SSB which set in at the transition point in the low temperature phase: ground state degeneracy, the appearance of a "massless" mode when the dynamics is invariant under a continuous symmetry, and the occurrence of a "massive" mode characterizing the rigidity of the order parameter.

In the absence of external magnetic fields and of surface effects, a ferromagnetic substance below the Curie point displays a global orientation of the magnetization, while the dynamics of the system is clearly rotation invariant, namely, the Hamiltonian of the system is invariant under the full rotation group. This is SSB.

A ferromagnetic system is composed of microscopic atomic magnets (in simplified models such as the Heisenberg model these are spin $1 / 2$ objects) whose interactions tend to orient neighboring ones parallel to each other. No global orientation appears at high temperature where the disordering thermal motion dominates. Below a critical "Curie temperature" energy considerations dominate and the system picks up a global magnetization. The parallel orientation of neighboring magnets propagates, ending up in a macroscopic magnetization. This selects a direction, which for an infinite isolated ferromagnet is arbitrary. It is easily proven that for an infinite system any pair of possible orientations define orthogonal ground states and any local excitations on top of these ground states are also orthogonal to each other. Thus the full Hilbert space of the system becomes split into an infinity of disjoint Hilbert spaces. This is ground state degeneracy.

The effective thermodynamical potential $V$ whose minimum yields the magnetization in the absence of an external

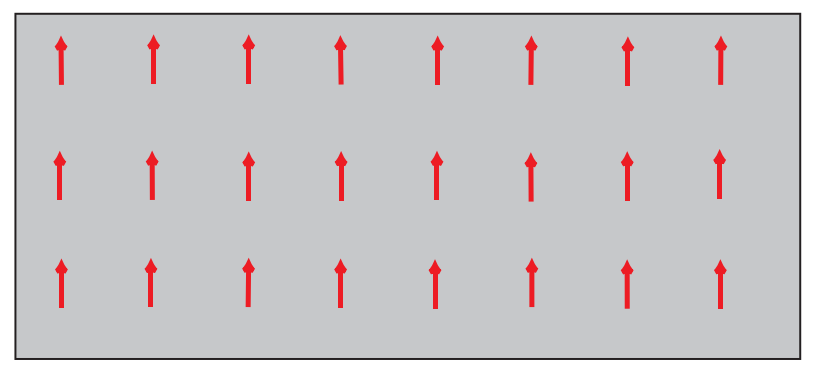

FIG. 2 (color). The ground state of a ferromagnet. 

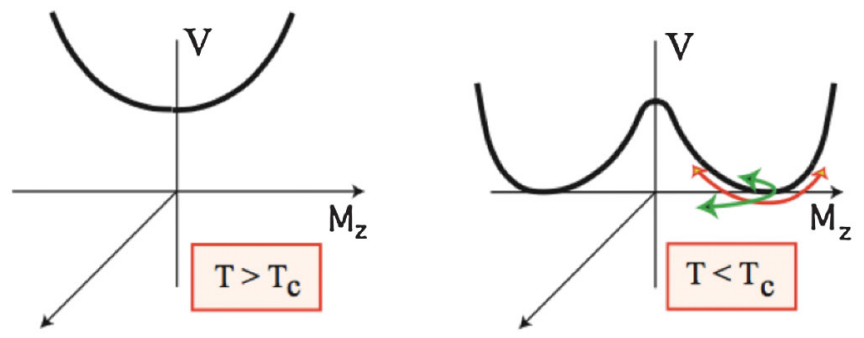

FIG. 3 (color). Effective thermodynamical potential of a ferromagnet above and below the Curie point.

magnetic field is depicted in Fig. 2. Above the Curie point $T_{c}$ the magnetization $\vec{M}$ vanishes. Below the Curie point in a plane $V M_{z}$ the potential develops in a plane $V M_{z}$ a double minimum which generates a valley in the $M_{x}, M_{y}$ directions. Each point of the valley defines one of the degenerate ground states with the same $|\vec{M}|$.

At a given minimum, say, $\vec{M}=M^{z} \overrightarrow{1}_{z}$, the curvature of the effective potential measures the inverse susceptibility which determines the energy for infinite wavelength fluctuations. This is the analog of mass in relativistic particle physics. The inverse susceptibility is zero in directions transverse to the order parameter and positive in the longitudinal direction. One thus obtains from the transverse susceptibility a massless transverse mode characteristic of broken continuous symmetry: these are the "spin waves" whose quantum constituents are interacting bosons called "magnons." The longitudinal susceptibility yields a (possibly unstable) massive longitudinal mode which corresponds to fluctuations of the order parameter. In contradistinction to the massless mode which exists only in continuous SSB for which there is a valley, the massive mode is present in any SSB, continuous or discrete and measures the rigidity of the ordered structure.

The structure of Fig. 3 is common to many second order phase transitions and leads to similar consequences. However in superconductivity a new phenomenon occurs. The quantum phase symmetry is broken by a condensation of electron pairs bounded by an attractive force due to phonon exchange in the vicinity of the Fermi surface. The condensation leads to an energy gap at the Fermi surface. For neutral superconductors, this gap would host a massless mode and one would recover the general features of SSB. But the presence of the long range Coulomb interactions modifies the picture. The massless mode disappears: it is absorbed in electron density oscillations, namely, in the massive plasma mode. As will be apparent later, this is a precursor of the BEH mechanism (Anderson, 1958, 1963; Nambu, 1960a).

\section{B. Spontaneous symmetry breaking in field theory}

Spontaneous symmetry breaking was introduced in relativistic quantum field theory by Nambu in analogy with the Bardeen-Cooper-Schrieffer (BCS) theory of superconductivity (Nambu, 1960a). The problem studied by Nambu (1960b) and Nambu and Jona-Lasinio (1961a), 1961b) is the spontaneous breaking of the $U(1)$ symmetry of massless fermions resulting from the arbitrary relative (chiral) phase between their decoupled right and left constituent neutrinos. Chiral invariant interactions cannot generate a fermion mass in perturbation theory but may do so from a (nonperturbative) fermion condensate: the condensate breaks the chiral symmetry spontaneously. Nambu (1960b) showed that such spontaneous symmetry breaking is accompanied by a massless pseudoscalar. This is interpreted as the chiral limit of the (tiny on the hadron scale) pion mass. Such interpretation of the pion constituted a breakthrough in our understanding of strong interaction physics. The massless pseudoscalar is the fieldtheoretic counterpart of the massless spin-wave mode in ferromagnetism. In the model of Nambu and Jona-Lasinio (1961a, 1961b) it is shown that SSB also generates a massive scalar boson which is the counterpart of the massive mode measuring in phase transitions the rigidity of the order parameter in the spontaneously broken phase.

The significance of the massless boson and of the massive scalar boson occurring in SSB is well illustrated in a simple model devised by Goldstone (1961). The potential $V\left(\phi_{1}, \phi_{2}\right)$ depicted in Fig. 4 has a rotational symmetry in the plane of the real fields $\left(\phi_{1}, \phi_{2}\right)$, or equivalently is invariant under the $U(1)$ phase of the complex field $\phi=\left(\phi_{1}+i \phi_{2}\right) / \sqrt{2}$. This symmetry is spontaneously broken by the expectation value $\langle\phi\rangle$ of the $\phi$ field acquired at a minimum of the potential in some direction of the $\left(\phi_{1}, \phi_{2}\right)$ plane, say $\left\langle\phi_{1}\right\rangle$. Writing $\phi=\langle\phi\rangle+\varphi$

$$
\begin{gathered}
\phi_{1}=\left\langle\phi_{1}\right\rangle+\varphi_{1}, \\
\phi_{2}=\varphi_{2}
\end{gathered}
$$

For small $\varphi_{1}$ and $\varphi_{2}$ we may identify the quantum fluctuation $\varphi_{1}$ climbing the potential as the massive mode measuring the rigidity of the SSB ground state selected by $\left\langle\phi_{1}\right\rangle$, and the quantum fluctuation $\varphi_{2}$ in the orthogonal valley direction as the massless mode characteristic of a continuous SSB.

Their significance is illustrated in Figs. 5 and 6 depicting, respectively, classical $\varphi_{2}$ and $\varphi_{1}$ wave modes, on the classical background $\left\langle\phi_{1}\right\rangle$. The corresponding massless and massive bosons are the quantum constituents of these waves.

Figure 5(a) represents schematically a lowest energy state (a "vacuum") of the system: a constant nonzero value of the field $\phi_{1}=\left\langle\phi_{1}\right\rangle$ pervades space-time. Figure 5(b) depicts the excitation resulting from the rotation of half the fields in the $\left(\phi_{1}, \phi_{2}\right)$ plane. This costs only an energy localized near the surface separating the rotated fields from the chosen vacuum. SSB implies indeed that rotating all the fields would cost no energy at all: one would merely trade the initial chosen vacuum for an equivalent one with the same energy. This

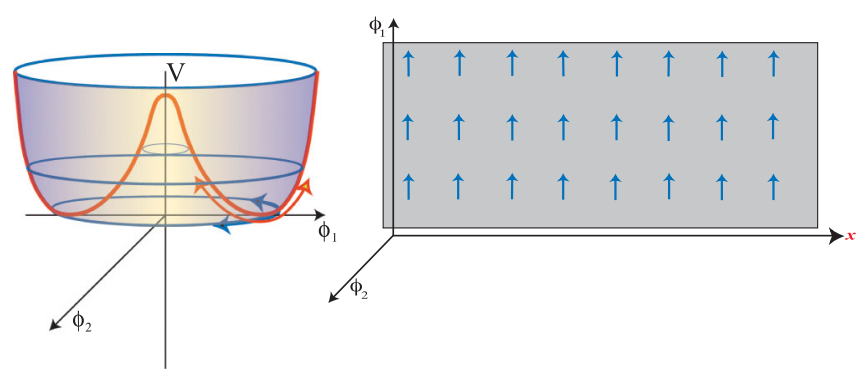

FIG. 4 (color). Spontaneous symmetry breaking in the Goldstone model. 


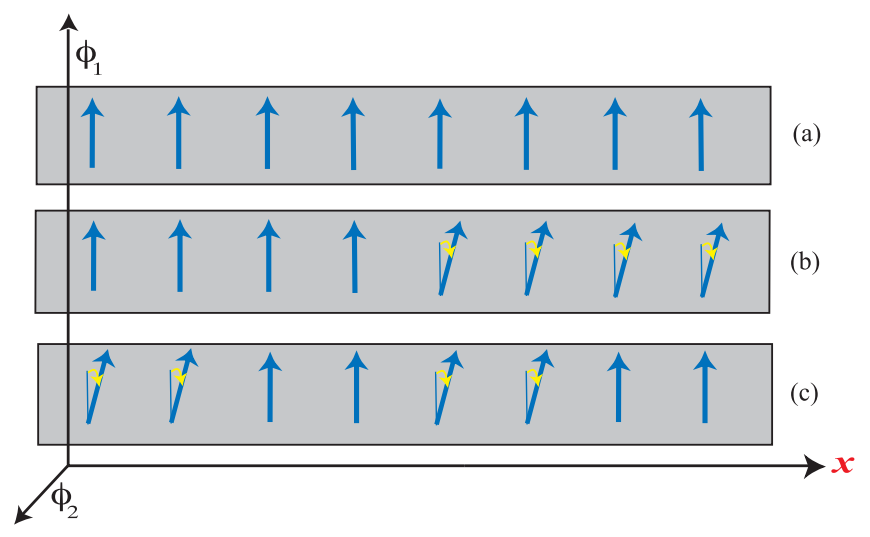

FIG. 5 (color). Massless Nambu-Goldstone mode $f_{2}$.

is the characteristic vacuum degeneracy of SSB. Figure 5(c) mimics a wave of $\varphi_{2}$. Comparing Fig. 5(c) with 5(b), we see that as the wavelength of the wave increases indefinitely, its energy tends to zero, and may be viewed as generating in that limit a motion along the valley of Fig. 4. Quantum excitations carried by the wave reach thus zero energy at zero momentum and the mass $m_{\varphi_{2}}$ is zero. Figure 5 can easily be generalized to more complex spontaneous symmetry breaking of continuous symmetries. Massless bosons are thus a general feature of such SSB already revealed by Nambu's discovery of the massless pion resulting from spontaneous chiral symmetry breaking (Nambu, 1960b). They will be labeled massless Nambu-Goldstone (NG) bosons. Formal proofs corroborating the above simple analysis can be found in the literature (Goldstone, Salam, and Weinberg, 1962).

Figure 6 depicts similarly a classical wave corresponding to a stretching of the vacuum fields. These excitations in the $\phi_{1}$ direction describe fluctuations of the order parameter $\left\langle\phi_{1}\right\rangle$. They are volume effects and their energy does not vanish when the wavelength becomes increasingly large. They correspond in Fig. 4 to a climbing of the potential. The quantum excitations $\varphi_{1}$ are thus now massive. These considerations can be again extended to more general SSB (even to discrete ones) to account for order parameter fluctuations. Lorentz invariance imposes that such massive excitations are necessarily scalar particles. They were also already present in Nambu and Jona-Lasinio (1961a, 1961b) and will be denoted in general as massive scalar bosons.

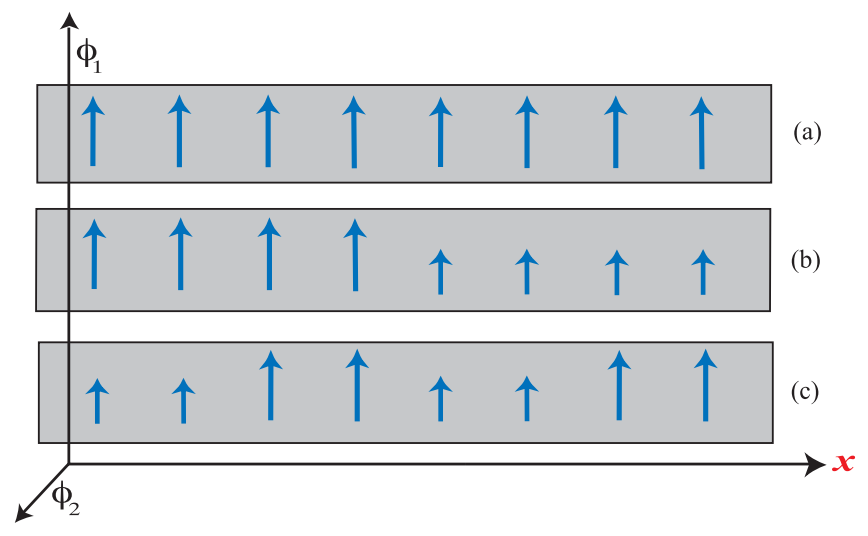

FIG. 6 (color). Massive scalar mode $f_{1}$.
To summarize, $\varphi_{2}$ describes massless bosons, $\varphi_{1}$ massive ones, and the "order parameter" $\left\langle\phi_{1}\right\rangle$ may be viewed as a condensate of $\varphi_{1}$ bosons.

\section{THE BEH MECHANISM}

The above considerations are restricted to spontaneous symmetry breaking of global continuous symmetries. Global means that the symmetry operations are independent of the space-time point $x$. For instance in the Goldstone model the global rotations of the fields in Fig. 5(a) in the $\left(\phi_{1}, \phi_{2}\right)$ plane by angles independent of the space-time point $x$ are symmetries of the theory (they describe motion in the valley of Fig. 4): these rotations cost no energy and simply span the degenerate vacua. We now discuss the fate of SSB when the global symmetry is extended to a local one.

\section{A. The fate of the Nambu-Goldstone boson and vector boson masses}

We extend the $U(1)$ symmetry of the Goldstone model from global to local. Thus the rotation angle in the $\left(\phi_{1}, \phi_{2}\right)$ plane in Fig. 5, or equivalently the rotation in the valley of Fig. 4, can now be chosen independently at each space-time point $(x)$ with no cost of energy and no physical effect. To allow such a feature, one has to invent a new field whose transformation would cancel the energy that such motion would generate in its absence. This is a "gauge vector field" $A_{\mu}$. It has to be a vector field to compensate energy in all space directions and it has to transform in a definite way under a rotation in the $\left(\phi_{1}, \phi_{2}\right)$ plane: this is called a gauge transformation and results in a large arbitrariness in the choice of the $A_{\mu}$ field corresponding to arbitrary "internal" rotations at different points of space. The consequence of this gauge symmetry is that the waves are polarized in directions perpendicular to their direction of propagation and that there quantum constituents have to be introduced as massless objects.

Local $U(1)$ symmetry is the simplest gauge field theory and is the symmetry group of quantum electrodynamics. In the local generalization (the gauging) of the Goldstone model, the introduction of the potential of Fig. 4 will deeply affect the "electromagnetic potential" $A_{\mu}$.

As in the Goldstone model of Sec. II.B, the SSB Yang-Mills phase is realized by a nonvanishing expectation value for $\phi=\left(\phi_{1}+i \phi_{2}\right) / \sqrt{2}$, which we choose to be in the $\phi_{1}$ direction. Thus

$$
\phi=\langle\phi\rangle+\varphi,
$$

with $\phi_{1}=\left\langle\phi_{1}\right\rangle+\varphi_{1}$ and $\phi_{2}=\varphi_{2}$. As previously, $\varphi_{2}$ and $\varphi_{1}$ appear to describe a NG massless boson and a massive scalar boson.

However, a glance at Fig. 5 depicting the NG mode immediately shows that Figs. 5(b) and 5(c) differ from Fig. 5(a) only by local rotations and hence in the local Goldstone model, they are just symmetry (or equivalently gauge) transformations. They cost no energy and therefore the NG boson has disappeared: the corresponding fluctuations in the valley are just a redundant (gauge transformed) description of the same gauge invariant vacuum. It is easy to see that this 
argument remains valid for any local symmetry and hence Nambu-Goldstone bosons do not survive the gauging of a global SSB to a local symmetry. The vacuum is no more degenerate and strictly speaking there is no spontaneous symmetry breaking of a local symmetry. The reason why the phase with nonvanishing scalar expectation value is often labeled SSB is that one uses perturbation theory to select at zero coupling with the gauge fields a scalar field configuration from global SSB; but this preferred choice is only a convenient one.

The disappearance of the NG boson is thus an immediate consequence of local symmetry. The above argument (Englert, 2005) was formalized much later (Elitzur, 1975) but formal proofs not directly based on the gauge invariance of the vacuum were already presented in 1964 (Guralnik, Hagen, and Kibble, 1964; Higgs, 1964b).

One may now understand in qualitative terms the consequence of the disappearance of the NG boson. Clearly, one does not expect that the degrees of freedom carried by the NG $\phi_{2}$ field could vanish. As the NG boson disappears because of its coupling to the gauge field, one expects that these degrees of freedom should be transferred to it. This can only occur by adding to the transverse polarization of the gauge field a longitudinal one. But such polarization is forbidden as mentioned earlier, for a massless field. Therefore the coupling of the would be NG boson to the gauge field must render the latter massive. This is the essence of the $\mathrm{BEH}$ mechanism.

These qualitative considerations can be made quantitative (Englert and Brout, 1964) by considering the Feynman graphs (time runs horizontally) describing the propagation of the $A_{\mu}$ gauge field in the vacuum with nonvanishing scalar field expectation value, say $\langle\phi\rangle \neq 0$. This propagation is depicted in lowest order in Fig. 7 (time runs horizontally) and the interaction of $A_{\mu}$ with the condensate $\langle\phi\rangle$ amounts to a "polarization" of the vacuum. The first graph shows the local interaction of the gauge field with the condensate while the second one gives a nonlocal interaction due to the propagation of a NG boson. Here $e$ is the coupling of the gauge vector to matter, $q_{\mu}$ is a four-momentum ( $q_{0}=$ energy; $\vec{q}=$ momentum), $q^{2}=q_{0}^{2}-\vec{q}^{2}$ and $g_{\mu \nu}$ has only nonzero values if $\mu \neq \nu: 1,-1,-1,-1$. The two graphs sum to

$$
\Pi_{\mu \nu}=\left(g_{\mu \nu}-q_{\mu} q_{\nu} / q^{2}\right) \Pi\left(q^{2}\right)
$$

where

$$
\Pi\left(q^{2}\right)=e^{2}\left\langle\phi_{1}\right\rangle^{2}
$$
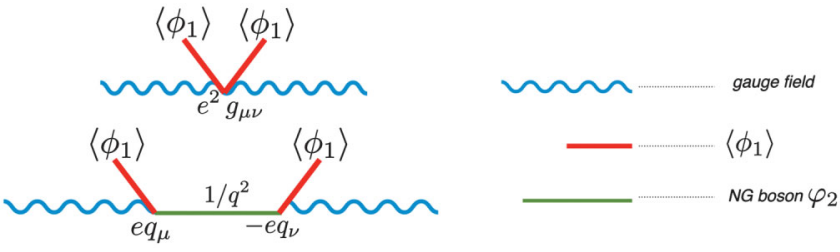

FIG. 7 (color). Interaction of the gauge field with the condensate.
The second factor of Eq. (3.2) does not vanish when $q^{2}=0$. In field theory this means that the gauge field has acquired a mass

$$
\left(M_{V}^{2}\right)=e^{2}\left\langle\phi_{1}\right\rangle^{2}
$$

The first factor describes the projection at $q^{2}=m_{V}^{2}$ of $g_{\mu \nu}$ on a three-dimensional space of polarizations, which, as explained in qualitative terms above, is required for a massive vector. Its transversality (i.e., its vanishing under multiplication by $q^{\mu}$ ) is characteristic of a "Ward identity" which expresses that the local gauge symmetry has not been broken and is identical to the analogous factor in quantum electrodynamics, an important fact that will be commented on in the following section.

The generalization of these results to more complicated symmetries. One gets (for real fields) a mass matrix

$$
\left(M_{V}^{2}\right)^{a b}=-e^{2}\left\langle\phi^{B}\right\rangle T^{a B C} T^{b C A}\left\langle\phi^{A}\right\rangle,
$$

where $T^{a B C}$ is a real antisymmetric generator coupled to a gauge field $A_{\mu}^{a}$ and $\left\langle\phi^{A}\right\rangle$ designates a nonvanishing expectation value.

In these cases, some gauge fields may remain massless. Consider for instance instead of the invariance of the Goldstone model on a circle in the plane $\left(\phi_{1}, \phi_{2}\right)$, an invariance on a sphere in a three-dimensional space $\left(\phi_{1}, \phi_{2}, \phi_{3}\right)$ broken by $\left\langle\phi_{1}\right\rangle \neq 0$. There are now three gauge fields associated with the rotations on the sphere, and while $A_{\mu}^{2}$ and $A_{\mu}^{3}$ acquire mass $A_{\mu}^{1}$ remains massless. This can be understood in the following way: rotation generators around the directions 2 and 3 would move $\left\langle\phi_{1}\right\rangle$ if the symmetry were global and would thus give rise, as in Fig. 5, to NG bosons; their degrees of freedom are transferred in local symmetries to the massive gauge vector fields $A_{\mu}^{2}$ and $A_{\mu}^{3}$, providing their third degree of polarization. The expectation value $\left\langle\phi_{1}\right\rangle$ is not affected by rotation generators around direction 1 and does not generate in the global symmetry case NG bosons and hence the corresponding $A_{\mu}^{1}$ remains massless.

Thus the BEH mechanism can unify in the same theory long and short range interactions by leaving unbroken a subgroup of symmetry transformation (e.g., rotation around direction 1) whose corresponding gauge fields remain massless.

\section{B. The fate of the massive scalar boson}

A glance at Fig. 5 shows that the stretching of (classical) scalar fields is independent of local rotations of the $\phi$ field in the $\left(\phi_{1}, \phi_{2}\right)$ plane. This translates the fact that the modulus of the $\phi$ field is gauge invariant. Hence the scalar bosons survive the gauging and their classical analysis is identical to the one given for the Goldstone model in Sec. II.B. The coupling of
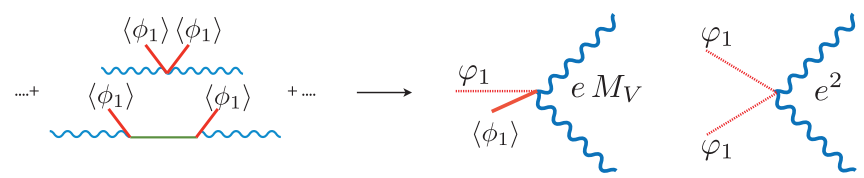

FIG. 8 (color). Coupling of the scalar boson $f_{1}$ to massive gauge bosons. 


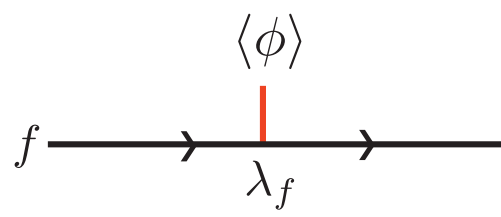

FIG. 9 (color). Mass generation $m_{f}=\lambda_{f}\langle\phi\rangle$ from a coupling $\lambda_{f}$ of fermions to the scalar field $\phi$.

the scalar boson $\varphi_{1}$ to the massive gauge bosons follows from the Fig. 7, by viewing the Feynman diagrams with time going from top to bottom and using Eq. (3.1). One gets the two vertices of Fig. 8 where the heavy wiggly lines on the right-hand side represent the massive gauge propagators. The vertex couplings follow from Eq. (3.4).

\section{Fermion masses}

Let us couple the Yang-Mills fields to massless fermions in a way that respects the Yang-Mills symmetry. This coupling preserves the chiral symmetry of the massless fermions and fermion mass requires SSB. In the Nambu theory of spontaneous breaking of chiral symmetry, this gives rise to $\mathrm{NG}$ bosons which are here eaten up by massive gauge fields. This can be done by suitable couplings of the scalar fields whose expectation value breaks the symmetry. Mass generation for fermions is depicted in Fig. 9.

\section{Why is the mechanism needed?}

Equation (3.2) expresses that the mass generation from the $\mathrm{BEH}$ mechanism does not destroy the local symmetry, in contradistinction to a mass term introduced by hand $a b$ initio. This equation remains valid at higher orders in perturbation theory and has the same form as the polarization in quantum electrodynamics. As in the latter case, it implies that in covariant gauges the gauge vector boson propagator tames the quantum fluctuations, and suggests therefore that the theory is renormalizable (Englert, Brout, and Thiry, 1966a; Englert, 1968). However, it is a highly nontrivial matter to prove that it does not introduce contributions from unphysical particles and it is therefore a very difficult problem to prove quantum consistency to all orders. That this is indeed the case has been proven by 't Hooft and Veltman ('t Hooft, 1971; 't Hooft and Veltman, 1972) [see also Lee and Zinn-Justin (1972a, 1972b, 1972c).

The quantum consistency of the BEH mechanism is the basic reason for its success. Precision experiments predicted and were indeed verified. The quantum consistency played a critical role in the analysis of the production of the scalar boson at the LHC and of its decay products, leading to the confirmation of the detailed validity of the mechanism.

\section{E. Dynamical symmetry breaking}

The symmetry breaking giving mass to gauge vector bosons may also arise from a fermion condensate. This is labeled as dynamical symmetry breaking. If a spontaneously broken global symmetry is extended to a local one by introducing gauge fields, the massless NG bosons disappear as previously from the physical spectrum and their absorption by gauge fields renders these massive. In contradistinction with breaking by the scalar field condensate, it is very difficult in this way to give mass in a renormalizable theory simultaneously to both gauge vector fields and fermions.

\section{F. The electroweak theory and the standard model}

The most impressive success of the BEH mechanism is the electroweak theory for weak and electromagnetic interactions applied to all particles of the standard model. These encompass all known particles. These are (a) the fermions which are listed in Fig. 10, (b) $\gamma$ and $W^{+}, W^{-}, Z$, the gauge vector bosons transmitting the electromagnetic and the weak interactions, (c) eight "gluons," the gauge vectors bosons of the "color group" SU(3) mediating the strong interactions, and, last but not least, (d) one massive scalar boson which was recently discovered and identified as the scalar predicted by the $\mathrm{BEH}$ mechanism.

The first row in Fig. 10 contains the basic constituents of the proton and the neutron, namely, the electron, the tree up and down colored quarks building the proton and neutron bonded by the gluons, to which is added the electron neutrino. The second (Glashow, Iliopoulos, and Maiani, 1970) and third rows (Kobayashi and Maskawa, 1973) were completed as predictions in the 1970s and verified afterward. Color was also introduced in the 1970s. The particles in the first and the second row are called leptons. To all fermions of the table, one must of course also add their antiparticles.

All the fermions are chiral and their chiral components have different group quantum numbers. Hence they are, as the gauge vector bosons, massless in absence of the $\mathrm{BEH}$ mechanism, i.e., in absence of the scalar condensate. The condensate $\langle\phi\rangle \neq 0$ gives mass to the $W^{+}, W^{-}, Z$ bosons and to all fermions except the three chiral neutrinos which have no opposite chirality counterpart in the conventional standard model. The photons and the gluons remain massless but the latter become short range due in the conventional description to a highly nonperturbative vacuum (resulting from a mechanism somehow dual to the $\mathrm{BEH}$ mechanism).

The discovery of the $Z$ and $W$ bosons in 1983 and the precision experiments testing the quantum consistency of the standard model established the validity of the mechanism, but it was still unclear whether this was the result of a dynamical

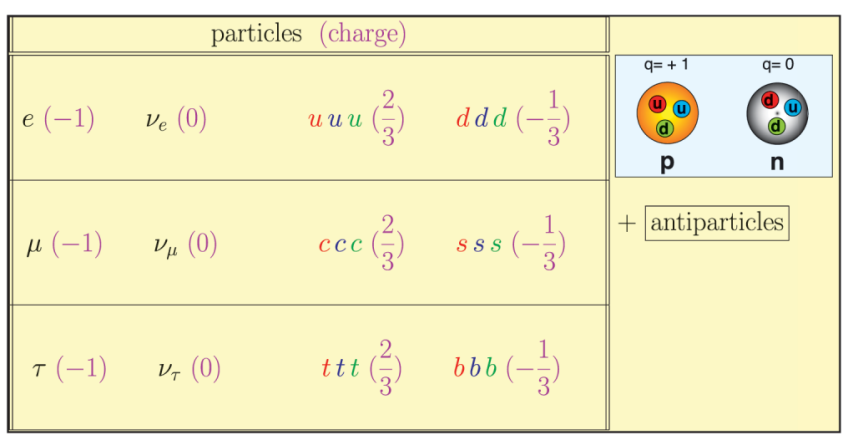

FIG. 10 (color). Fermion constituents of the standard model. 
symmetry breaking or of a particle identifiable as an elementary boson at the energy scale considered.

\section{THE DISCOVERY}

In the standard model, there is one real massive scalar boson $\varphi$ (also labeled $H$ ). It couples to the massive $W$ and $Z$ bosons. This follows from Fig. 8 and the couplings are depicted in Fig. 11(a). Its coupling to elementary fermions similarly follows from the couplings in Fig. 9 as shown in Fig. 11(b). The coupling to the massless photons is a genuine quantum effect involving loops, even in lowest order, as indicated in Fig. 12.

The LHC site circling under the French-Swiss borders is schematically indicated in the picture of Fig. 13. The $27 \mathrm{~km}$ circular tunnel containing two opposite beams of protons surrounded by guiding superconducting electromagnets cooled by superfluid helium is pictured in Fig. 14.

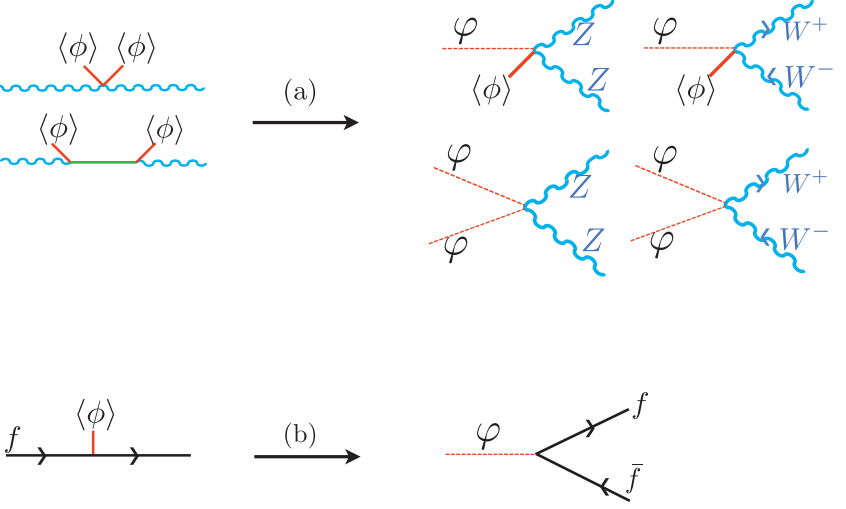

FIG. 11 (color). Coupling of the scalar boson $f$ to massive gauge bosons and to elementary fermions.

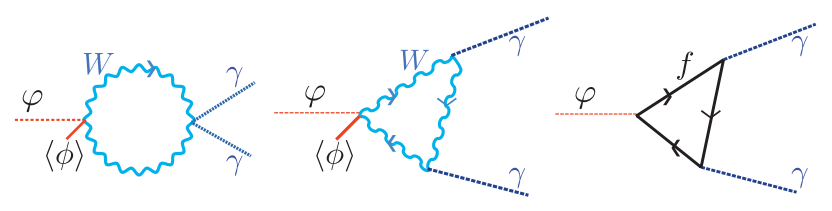

FIG. 12 (color). Coupling of the scalar boson $f$ to photons.

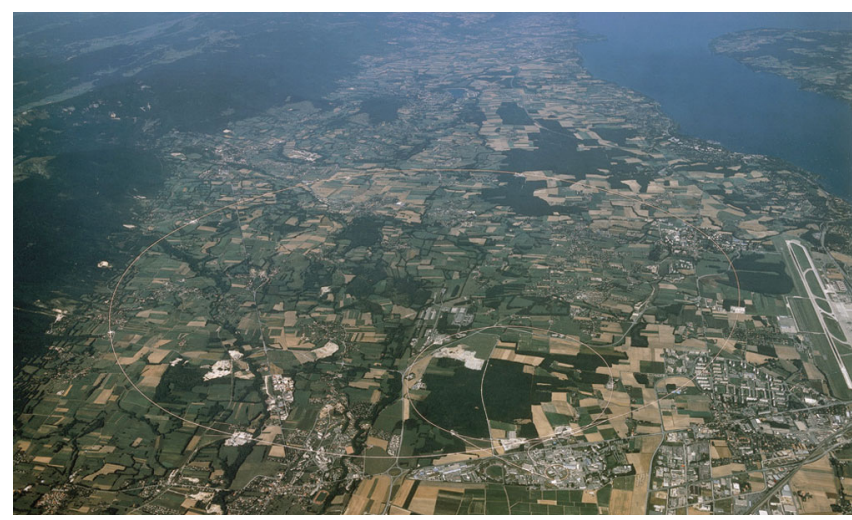

FIG. 13 (color). Schematic location of the LHC.

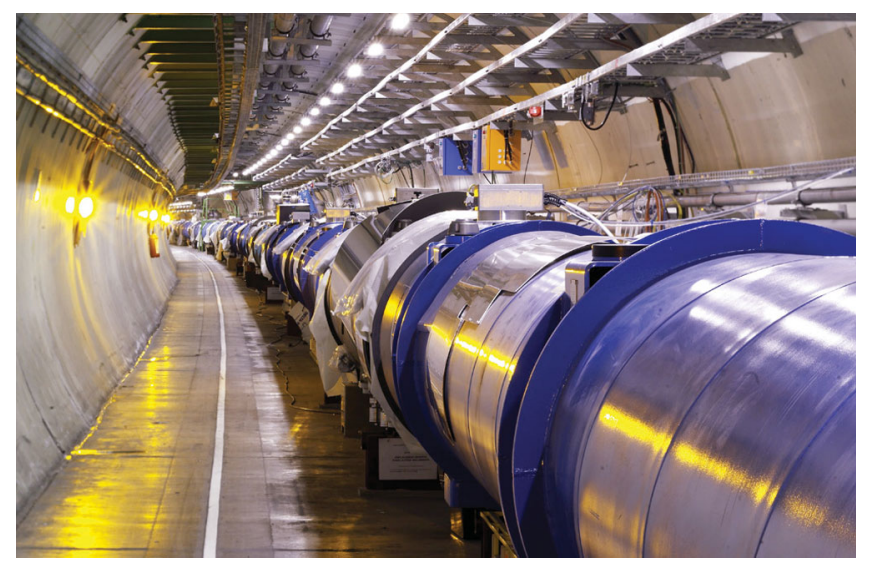

FIG. 14 (color). The LHC dipole magnets.

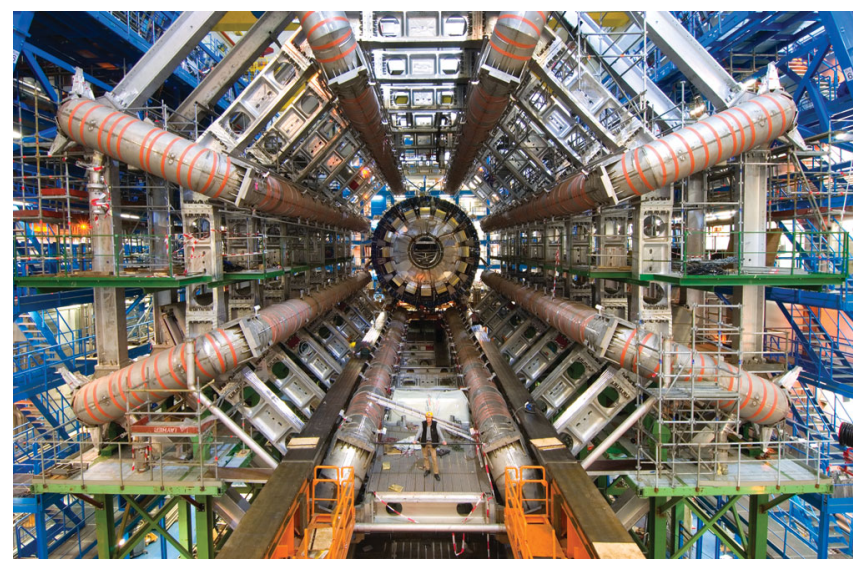

FIG. 15 (color). The ATLAS detector.

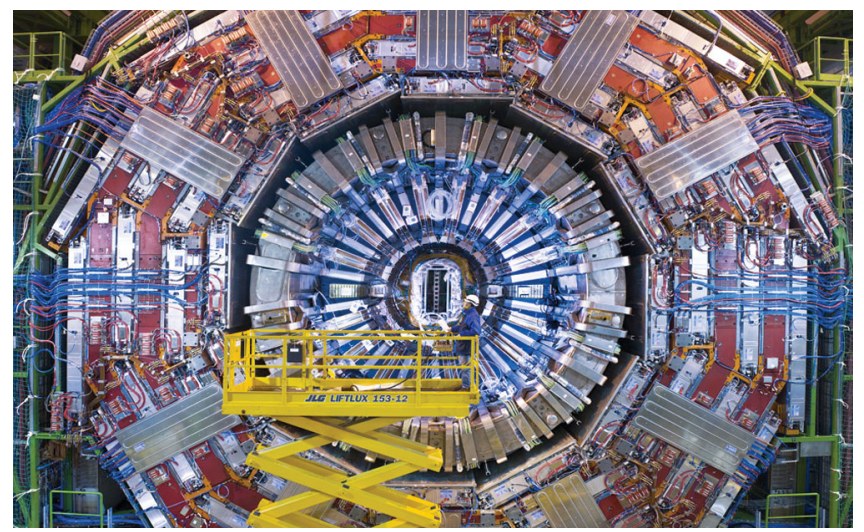

FIG. 16 (color). The CMS detector.

Figures 15 and 16 are pictures of the ATLAS and CMS detectors at diametrical opposite sites of the tunnel. There collisions occur and were used primarily to detect and identify the scalar boson of the standard model (and possibly other ones). At the end of 2012 proton-proton collisions occurred at the rate of nearly $10^{9} \mathrm{~s}^{-1}$ and the proton energy reached $8 \mathrm{TeV}$. At these energies, all quarks of Fig. 10 and the gluons connecting them may contribute to the production of the scalar boson. The leading production processes are represented in Fig. 17. 


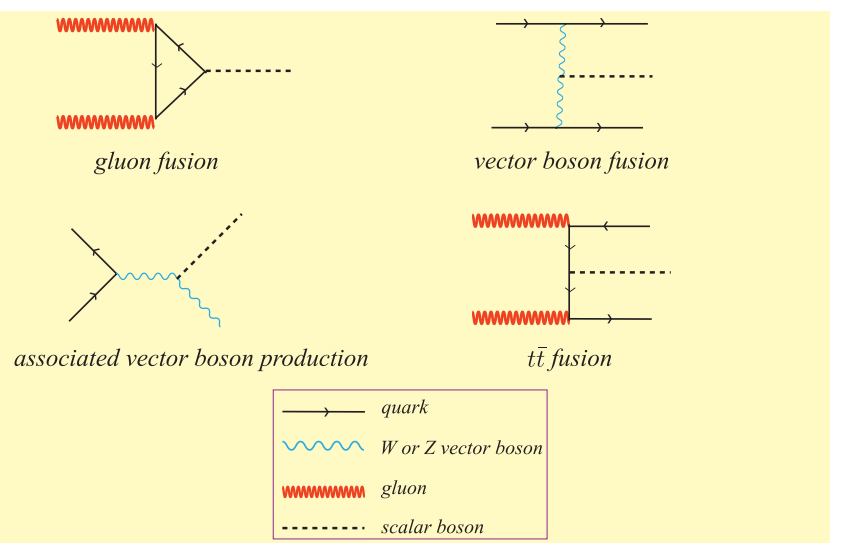

FIG. 17 (color). Production of the standard model scalar.

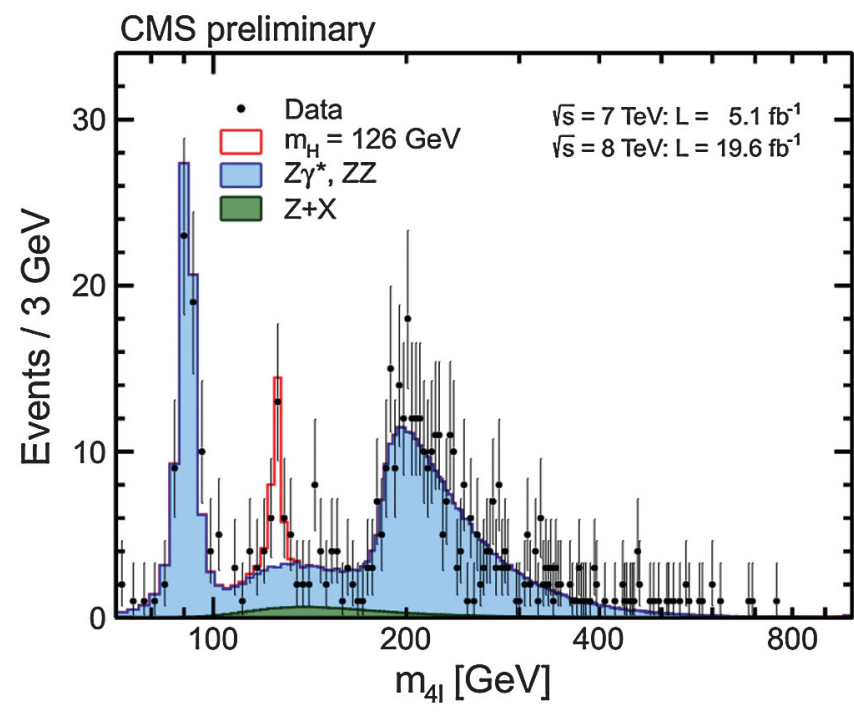

FIG. 18 (color). Decay of the scalar boson into 4 leptons from two Zs.

As an example of the data gathered by CMS and ATLAS, Fig. 18 gives the data obtained by the CMS group of observed decays into 4 leptons at the end the 2012 run. The blue area is the expected background, namely, those decays which would follow from the standard model if, at given total mass, there would be no contribution from the scalar boson. The red curve measures the contribution that could be due to the scalar decaying into two $Z$ vector bosons which further decay into leptons, as was confirmed by further analysis. Note that one of the $Z$ is real but the other is "virtual," meaning that this decay is forbidden by energy conservation but may contribute in the quantum theory. Consideration of other decay channels and spin analysis show that the particle detected is consistent with the standard model scalar with a mass $m_{H} \simeq 125 \mathrm{GeV}$. The absence of new particles at comparable energies, as well as the success of the Feynman graph analysis including loops, points toward an elementary particle, at least up to the energy range considered. This is the first elementary spin zero particle ever detected. It raises the interesting possibility of supersymmetry broken at attainable energies, although there is no indication of it so far.

The elementary character of the scalar already eliminates many dynamical models of symmetry breaking and raises interesting possibilities for extrapolation beyond presently known energies, up to those close to the Planck scale where quantum gravity effects might play a dominant role. The analyses of these speculations are beyond the scope of this talk.

\section{REFERENCES}

Anderson, P. W., 1958, "Random-phase approximation in the theory of superconductivity," Phys. Rev. 112, 1900.

Anderson, P. W., 1963, "Plasmons, gauge invariance, and mass," Phys. Rev. 130, 439.

Elitzur, S., 1975, "Impossibility of spontaneously breaking local symmetries," Phys. Rev. D 12, 3978.

Englert, F., 1968, in "Fundamental Problems in Elementary Particle Physics," Proceedings of the Fourteenth Solvay Conference on Physics (John Wiley, New York), p. 18.

Englert, F., 2005, "Broken symmetry and Yang-Mills theory," in 50 Years of Yang-Mills Theory, edited by G.'t Hooft (World Scientific, Singapore), pp. 65-95.

Englert, F., and R. Brout, 1964 "Broken symmetry and the mass of gauge vector mesons," Phys. Rev. Lett. 13, 321.

Englert, F., R. Brout, and M. Thiry, 1966a, "Vector mesons in presence of broken symmetry," Il Nuovo Cimento A 43A, 244.

Glashow, S. L., J. Iliopoulos, and L. Maiani, 1970, "Weak interactions with lepton-hadron symmetry," Phys. Rev. D 2, 1285.

Goldstone, J., 1961, "Field theories with "superconductor" solutions," Il Nuovo Cimento 19, 154.

Goldstone, J., A. Salam, and S. Weinberg, 1962, "Broken symmetries," Phys. Rev. 127, 965.

Guralnik, G. S., C. R. Hagen, and T. W. B. Kibble, 1964, "Global conservation laws and massless particles," Phys. Rev. Lett. 13, 585.

Higgs, P. W., 1964a, "Broken symmetries and the masses of gauge bosons," Phys. Rev. Lett. 13, 508.

Higgs, P. W., 1964b, "Broken symmetries, massless particles and gauge fields," Phys. Lett. 12, 132.

Kobayashi, M., and T. Maskawa, 1973, "CP Violation in the renormalizable theory of weak interaction," Prog. Theor. Phys. 49, 652.

Landau, L. D., 1937, "On the theory of phase transitions I," Phys. Z. Sowjetunion 11, 26 [JETP 7, 19 (1937)].

Lee, B. W., and J. Zinn-Justin, 1972a, "Spontaneously broken gauge symmetries. I. Preliminaries,” Phys. Rev. D 5, 3121.

Lee, B. W., and J. Zinn-Justin, 1972b, "Spontaneously broken gauge symmetries. II. Perturbation theory and renormalization," Phys. Rev. D 5, 3137.

Lee, B. W., and J. Zinn-Justin, 1972c, "Spontaneously broken gauge symmetries. III. Equivalence,” Phys. Rev. D 5, 3155.

Nambu, Y., 1960a, "Quasi-particles and gauge invariance in the theory of superconductivity," Phys. Rev. 117, 648.

Nambu, Y., 1960b, "Axial vector current conservation in weak interactions," Phys. Rev. Lett. 4, 380.

Nambu, Y., and G. Jona-Lasinio, 1961a, "Dynamical model of elementary particles based on an analogy with superconductivity. I," Phys. Rev. 122, 345.

Nambu, Y., and G. Jona-Lasinio, 1961b, "Dynamical model of elementary particles based on an analogy with superconductivity. II," Phys. Rev. 124, 246.

't Hooft, G., 1971, "Renormalizable Lagrangians for massive Yang-Mills fields," Nucl. Phys. B35, 167.

't Hooft, G., and M. Veltman, 1972, "Regularization and renormalization of gauge fields," Nucl. Phys. B44, 189. 